\title{
Factores de diversidad y fluctuación poblacional de insectos asociados al cultivo de piña (Ananas comosus L. Merril) en Ticuantepe, Nicaragua
}

\section{Diversity factors and population variation of insects associated to Pineapple (Ananas comosus L. Merril) in Ticuantepe, Nicaragua}

\section{Carlos Mairena Vásquez ${ }^{1}$, Edgardo Jiménez-Martínez ${ }^{2}$}

${ }^{1}$ MSc en Sanidad Vegetal, UNA, Instituto de protección y sanidad agropecuaria (IPSA)

${ }^{2} \mathrm{PhD}$ en Entomología, Universidad Nacional Agraria (UNA), profesor titular, edgardo.jimenez@ci.una.edu.ni / Tel: 505-22632609.

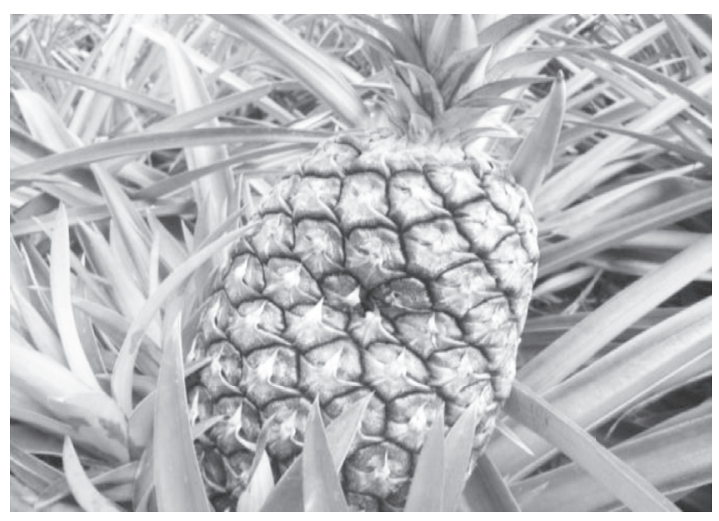

\section{RESUMEN}

La piña (Ananas comosus L. Merril) está entre los cultivos de mayor aceptación y de mayor demanda en Nicaragua y nivel regional e internacional, siendo a nivel nacional una de las frutas de mayor producción. Este estudio se hizo con el objetivo de identificar los principales insectos asociados a la piña, su diversidad, riqueza y abundancia, además de describir su fluctuación poblacional y el hábito alimenticio. La investigación se realizó en el período de marzo a septiembre del 2014, en la zona piñera del Municipio de Ticuantepe. El estudio se desarrolló en seis fincas piñeras donde se ubicaron trampas de caída libre (Pitfall traps) para la captura de insectos rastreros, trampas de galones de plástico con melaza para la captura de insectos voladores y en cada sitio se colectó manualmente insectos del follaje frutos y suelo. En el estudio se encontró que las principales familias de insectos asociados al cultivo fueron Formicidae, Scarabaeidae, Pseudococcidae, Elateridae, Noctuidae, Nymphalidae Cerambicidae y Lycaenidae. La diversidad insectil se estimó utilizando el índice de diversidad Shannon-Weaver, la familia con mayor índice de diversidad fue Formicidae, En cuanto a la riqueza de insectos se encontraron cincuenta y un familias y ochenta y cuatro géneros, siendo los más numerosos pertenecientes a las familias; Formicidae, Scarabaidae, Tenebrionidae y Pentatomidae. En lo que respecta a la fluctuación poblacional, las familias de insectos que presentaron mayor incidencia fueron: Formicidae, Scarabaidae y Pseudococcidae, las fluctuaciones más altas de insectos ocurrieron en los meses de abril, mayo y julio, entre los principales hábitos alimenticios de las especies insectiles asociados al cultivo de la piña se encontraron, defoliadores, plagas del suelo, succionadores de floemas, barrenadores de corteza vegetal, polinizadoras, depredadores, parasitoides y degradadores de materia orgánica.

Palabras clave: piña, insectos, diversidad, abundancia, riqueza, fluctuación poblacional.

\begin{abstract}
The pineapple (Ananas comosus L. Merril) is among others fruits, one of the most accepted crops in Nicaragua, regionally and internationally, being nationally an increased production fruits. The objectives of this study were, identification, abundance, richness, diversity, population dynamics and eating habits of the main insect families associated to pineapple. This study was conducted in the period from March to September 2014, in the pineapple area of the Municipality of Ticuantepe, The study was conducted in six pineapple farms where three Pitfall traps for catching crawling insects, three plastic gallons with molasses traps for catching flying insects were placed, in addition, insects on fruits, foliage and soil were manually collected. This research found that the main families found associated to pineapple were; Formicidae, Scarabaeidae, Pseudococcidae, Elateridae, Noctuidae, Nymphalidae, Cerambicidae and Lycaenidae. The insect diversity was estimated using the Shannon-Weaver diversity index, the family with the highest diversity was Formicidae, regarding the insect richness, fifty one families and eighty four genus were found, the most numerous families were; Formicidae, Scarabaidae, Tenebrionidae and Pentatomidae, in regard to the population dynamics, insect families with the highest incidence were: Formicidae, Scarabaidae and Pseudococcidae, higher insect fluctuations occurred in the months of April, May and July, the main eating habits of insect associated to pineapple were found as, defoliators, soil pests, sniffers phloem, bark borers plant, pollinators, predators, parasitoids and degraders organic matter.
\end{abstract}

Key words: Pineapple, insects, diversity, abundance, richness, population fluctuation.
Recibido: 17 de noviembre 2016 Aceptado: 25 de enero del 2017

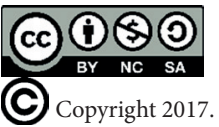

Los artículos de la revista La Calera de la Universidad Nacional Agraria, Nicaragua, se comparten bajo términos de la licencia Creative Commons: Reconocimiento, No Comercial, Compartir Igual. Las autorizaciones adicionales a las aquí delimitadas se pueden obtener en el correo freddy.aleman@ci.una.edu.ni Universidad Nacional Agraria 
$\mathrm{L}$

a piña (Ananas comosus L. Merril) es originaria de Suramérica, específicamente del área fronteriza entre Brasil y Paraguay, esta es una planta que pertenece a la familia de las Bromeliaceae, fue domesticada por los indígenas en tiempos precolombinos y llevada a América Central, México y las Antillas (Bonilla, 1992, INTA, 2001). La piña por primera vez fue introducida a Nicaragua desde honduras en 1958 con la variedad Monte Lirio y en el año 1968 fue introducida la variedad Cayena Lisa desde Costa Rica (FDA, 1992). La piña como fruta tropical, tiene alta demanda en el mercado de nuestro país y los frutos se consumen frescos o en conservas. En cuanto a los desechos vegetales (tallos, hojas, y cáscara de fruta) pueden ser aprovechados para alimentación animal (INTA, 2001).

Según ATC (2011) indica que en la zona de Ticuantepe hay presencia de frutas durante todo el año, la época principal de floración natural de la piña ocurre a partir de los primeros días de diciembre hasta los primeros días de febrero; que es la primera floración, esto es debido principalmente al acortamiento del día y por las bajas temperaturas durante la noche. Una segunda floración de muy poca importancia ocurre en agosto y septiembre por las altas nubosidades existentes. La mayor producción de frutos se concentra casi en su totalidad en los meses de junio, julio y agosto.

Entre los insectos identificados y que representan mayor problema desde el punto de vista fitosanitario en el cultivo de la piña están; Gallina ciega (Phyllophaga sp), barrenador del fruto (Thecla basilides) y Cochinillas (Dysmococcus brevipes) (INTA, 2001). Los factores agroecológicos y los mecanismos de regulación interno de la plantas de piña, influenciados directamente por el cambio físico del ambiente, juegan un papel determinante dentro del manejo preventivo de plagas. La susceptibilidad de la planta ante estos agentes fitófagos y patógenos, está muy relacionada a la fenología del cultivo, a las condiciones ambientales y al manejo de cultivo (OIRSA, 1999). Por eso es de esencial importancia tener conocimiento de las etapas fenológicas del cultivo y de la relación que existe entre plagas, con las etapas del cultivo, lo que permitirá organizar todas las medidas fitosanitarias esenciales, para el control preventivo de las plagas. En Nicaragua, se conocen algunos insectos asociados a piña; sin embargo, se desconoce la fluctuación poblacional de estos insectos, su diversidad, riqueza y abundancia. Es importante para los productores de piña de Nicaragua conocer algunos factores de diversidad y fluctuación poblacional de insectos asociados a la piña, con esta información, los productores pueden diseñar mejores prácticas de manejo integrado de plagas en su cultivo.

\section{MATERIALES Y MÉTODOS}

Localización geográfica del estudio. Este estudio se realizó entre los meses de marzo a septiembre del 2014, en seis fincas piñeras del municipio de Ticuantepe, Managua, las fincas fueron Los Caleros, Las Perlas, San Felipe La Francia, Los Ríos y El Edén, todas ellas localizadas en comunidades del municipio antes mencionado, el cual está ubicado entre las coordenadas $12^{\circ} 01^{\prime} 00^{\prime \prime}$ de latitud norte y $86^{\circ} 12^{\prime} 00^{\prime \prime}$ lon- gitud oeste, a una altura que oscilan entre 139 a $874 \mathrm{msnm}$, con temperaturas medias anuales de entre 22 y $29^{\circ} \mathrm{C}$ y precipitaciones promedios anuales de $900 \mathrm{~mm}$ y $60 \%$ de humedad relativa promedio (INIFON, 2009).

Descripción de las fincas. Las condiciones climáticas y la forma de manejo de las fincas evaluadas en el estudio eran similares y de producción convencional (altos insumos químicos), las variedades de piña cultivadas en las fincas seleccionadas fueron Monte Lirio y MD 2 con una edad promedio de los plantíos de dos años. Las áreas cultivadas con piña en las fincas evaluadas oscilan entre 0.15 a 14 hectáreas y están situadas a alturas entre los 336 a 436 metros sobre el nivel del mar (msnm), los suelos generalmente son de textura franco arenosa, la topografía del terreno va de moderadamente inclinado a moderadamente escarpado con pendientes entre $15 \%$ a $30 \%$.

Diseño experimental. El diseño del estudio consistió en la comparación de seis parcelas de piñas ubicadas en el Municipio de Ticuantepe. En cada finca se colocaron 6 trampas para captura de insectos; 3 trampas del tipo Pitfall Traps (trampas de caída libre) y 3 trampas del tipo galones de plástico de color blanco con agua y melaza, además se realizó conteo de plagas del suelo y de las partes aéreas de las plantas (hojas, tallo, inflorescencia y frutos). En las fincas se cuantificaron las poblaciones de insectos cada quince días.

\section{Metodología para el muestreo de insectos \\ Descripción del método de muestreo usando trampas de caída libre (Pitfall traps). Para la captura y colecta de insec-} tos rastreros se utilizó la metodología de (Rugama y López, 2011, Lacayo y Mayorga 2014 y Téllez y Jirón, 2014), la cual consistió en la utilización por finca de tres trampas de caída libre (Pitfall traps) ubicadas en el interior de la parcela y a una distancia de 10 metros entre trampas. Las trampas Pitfall traps consistían en panas plástica de $30 \mathrm{~cm}$ de diámetro de color blanco, colocadas al ras del suelo con capacidad de cuatro litros de agua, a las cuales se le agregaban cuatro gramos de detergente $\mathrm{Xedex}^{\circledR}(1 \mathrm{~g} /$ Litro de agua) y $250 \mathrm{cc}$ de refrigerante (Glicol de etileno) para vehículo para evitar evaporización del agua.

Los muestreos consistieron en la recolecta de todos los insectos encontrados caídos dentro de las panas, estos insectos se depositaban en viales entomológicos de 4.5 pulgadas de alto y de $3 \mathrm{~cm}$ de diámetro, conteniendo alcohol al $70 \%$, estos se rotularon con la fecha, nombre del productor y el número de sitio muestreado, todos los insectos encontrados en las panas se registraron en una hoja de muestreo.

Descripción del método de muestreo, utilizando trampas de galón plástico con agua y melaza. Para la captura y colecta de insectos voladores, según metodología usada por (Lacayo y Mayorga 2014 y Téllez y Jirón, 2014), se utilizaron trampas de galones blanco con melaza ubicadas en el interior de las parcelas de piña, utilizando tres trampas por finca, a una distancia de 10 metros entre trampas. Estos recipientes se colocaron horizontalmente y sujetadas a tutores a una altura 
de 30 centímetros por encima de la altura de las plantas de piña. Las trampas consistían en galones plásticos cortados en uno de sus costados, con capacidad de cuatro litros de agua, a la que se le adicionó 100 cc de melaza; lo que funcionó como atrayente por el olor, color y sabor dulce para insectos voladores, además se le adicionó dos litros de agua y $250 \mathrm{cc}$ de refrigerante (Glicol de etileno) para vehículo para evitar evaporización del agua.

La inspección a las trampas se realizó cada quince días, dándole mantenimiento, limpiándolas y cambiando la solución. Los insectos se colectaron en viales entomológicos con alcohol al $70 \%$ los que se etiquetaron y se identificaron en el laboratorio de entomología de la Universidad Nacional Agraria (UNA).

Descripción del método de muestreo en suelo y raíces. Para la observación y colecta de insectos plagas del suelo, a lo interno de la parcela se realizaron tres muestreos por finca cada quince días, donde se recolectaron estadios larvales y adultos de insectos, para ello se realizaron excavaciones en el suelo con dimensiones de 30x30x30 centímetros a una distancia de 10 metros entre excavación, En cada estación se revisaron las raíces de plantas, los insectos encontrados se depositaron en tubos viales con alcohol al 70\% y posteriormente trasladarlos al laboratorio de entomología.

Descripción del método de muestreo de observación a ramas, hojas y frutos de piña. Para la inspección de follaje, ramas, flores y frutos de piña, en cada finca se evaluaron treinta plantas al azar en tres estaciones, en cada estación se tomaron diez plantas en línea recta, donde se colectaron insectos que se encontraron sobre el follaje, tallos, inflorescencias y frutos, así mismo se colectaron partes de la planta $\mathrm{y}$ frutos con lesiones que posteriormente se trasladaron al laboratorio de entomología para su inspección. Todos los datos e información que se generó en las inspecciones y muestreos se llevaron en una hoja de registro.

Procesamiento de muestras e identificación de insectos en laboratorio. Las muestras de insectos recolectadas, fueron trasladadas al laboratorio de entomología de la UNA donde se realizó el montaje. Se tomaron los insectos de los viales entomológicos y se colocaron en platos petrix de $14 \mathrm{~cm}$ de ancho y $2 \mathrm{~cm}$ de largo para ser lavados en alcohol al 70\%, luego cada muestra de insectos se colocó en papel toalla para secar durante unos 30 minutos a temperatura ambiente, se contaron los insectos y se anotaron en una hoja de muestreo, Para el montaje de los insectos en las cajas entomológicas, se procedió a la utilización de gradillas entomológicas, pinzas, pinceles, alfileres entomológicos número 4.

En las cajas entomológicas se ordenaron cada insecto, rotulándolo con dos etiquetas, la primera etiqueta contenía la siguiente información (fecha, finca, número de trampas, país, departamento y colector), la segunda etiqueta llevaba el orden, familia, género y la especie a la que pertenece el insecto.

Todos los datos obtenidos se anotaron junto con la cantidad de insectos identificados en una hoja de registro. Los insectos se observaron con ayuda del estereoscopio y se realizó una revisión bibliográfica, para lograr una identificación final hasta el nivel de familia. Luego estos fueron trasladados al museo entomológico de la UNA, donde se realizaron verificaciones de especímenes; con las especies existentes en el museo y también se consultaron literaturas y libros relacionados a la taxonomía de insectos como (Borror y De Long, 1979, Hernández, 1992, King y Sanders, 1984, Maes, 2004). Además de la revisión de literatura específica como (Jiménez- Martínez y Rodriguez- Flores, 2014, Nunes y Dávila, 2004, Sáenz y De La Llana, 1990), se realizó una verificación preliminar de los especímenes. Se observaron las claves taxonómicas y se logró así la identificación final hasta nivel de género y en algunas ocasiones, especie para algunos insectos, las identificaciones preliminares estuvieron a cargo del técnico en entomología Alex Cerrato, responsable del museo entomológico de la UNA, la identificación final estuvo a cargo del Dr. Edgardo Jiménez Martínez.

Variables evaluadas. Las variables evaluadas fueron: número de insectos por finca, índice de diversidad Shannon Wiener, Aabundancia total de insectos encontrados por finca, y riqueza de insectos encontrados por finca.

Cálculo del índice de diversidad. Los índices empleados en la medición de la diversidad insectil fueron el de Shannon-Weaver, 1949, que varía de 0 a Logaritmo natural del número de especies, determinadas por el número de especies presentes en cada unidad de muestreo y basándose en la escala logarítmica escogida $\sum, \mathrm{H}^{\mathrm{ee}}=$ índice de Shannon - Weaver, $\ln =$ logaritmo natural, $\mathrm{pi}=\mathrm{ni} / \mathrm{N}$; donde $\mathrm{ni}=$ al número de individuos de cada especies y $\mathrm{N}=$ número total de individuos; este índice permitió cuantificar la biodiversidad específica en cada una de las unidades de muestreo. El otro índice utilizado fue el de Margalef que permitió estimar la biodiversidad de las unidades de muestreo, pero en base a la distribución numérica de los individuos de las diferentes especies en función del número de individuos existentes en la muestra analizada, este índice no considera el valor de importancia de las especies, solo se basa en el número de especies presentes, se lo obtuvo mediante la fórmula, donde $\mathrm{S}=$ número de especies y $\mathrm{N}=$ número total de individuos.

Cálculo de la abundancia. Para el cálculo de la abundancia se utilizó la metodología usada por Lacayo y Mayorga, 2014 y Téllez y Jirón, 2014, Esta abundancia se midió en números de insectos por finca, además se comparó porcentualmente la abundancia de insectos por familia encontrados en el cultivo de la piña entre las fincas evaluadas durante toda la etapa de muestreo. Para determinar si la abundancia de insectos por familia fue semejante en las diferentes unidades de muestreo, se empleó el índice de equidad de Pielou que se obtiene utilizando la fórmula, en donde $\mathrm{H}^{\text {ee }} \max =\ln (\mathrm{S})$. Esta misma metodología fue utilizada por Somarriba, 1999 y Vásquez et al., 2009, para medir abundancia insectil.

Cálculo de la riqueza de especies de insectos. El índice de riquezas fue calculado por medio del índice de Margalef, 1958. Donde $\mathrm{DMg}=\mathrm{S}-1 / \mathrm{Ln} \mathrm{N}$. Donde $\mathrm{S}=$ número de especies y $\mathrm{N}=$ número total de individuos. Riqueza es el número 
de especies por sitio de muestreo, o sea supone una relación entre el número de especies y el número total de individuos. La riqueza específica es la forma más sencilla de medir la biodiversidad, ya que se basa únicamente en el número de especies presentes, sin tomar en cuenta el valor de importancia de las mismas. Este método compara la riqueza de especies entre las muestras recogidas de diferentes hábitats (Moreno, 2001).

Análisis estadístico de los datos. Para el análisis de los datos se siguió la metodología utilizada por (Barrios et al., 2004, Urías et al., 2007, Rugama y López, 2011, Lacayo y Mayorga, 2014 y Téllez y Jirón, 2014), los cuales consisten en que después de colectados los datos de insectos, estos fueron arreglados por familias y por finca en una tabla de datos en EXCELL, luego cada variable fue comparada entre fincas, efectuando un análisis de varianza, seguido de una prueba de comparación por Tukey utilizando el programa de SAS (SAS, 2003). El nivel de significancia usado en el análisis fue de $(\mathrm{P}=0.05)$.

\section{RESULTADOS Y DISCUSIÓN}

Los principales órdenes, familias, géneros y especies de insectos encontrados en el cultivo de piña son presentados en el (cuadro 1).

Cuadro 1. Orden, familia, género, especie, nombre común y hábito alimenticio de insectos encontrados en el cultivo de la Piña en Ticuantepe, entre Marzo y Septiembre del 2014

\begin{tabular}{|c|c|c|c|c|}
\hline Orden & Familia & Especie & Nombre Común & Hábito Alimenticio \\
\hline Hymenoptera & Formicidae & Camponotus sp & Hormiga & Depredadora \\
\hline Hymenoptera & Formicidae & Solenopsis sp & Hormiga & Depredadora \\
\hline Hymenoptera & Formicidae & Pheidole sp & Hormiga & Depredadora \\
\hline Hymenoptera & Formicidae & Ectatomma sp & Hormigón & Defoliador \\
\hline Hymenoptera & Formicidae & Atta cephalotes & Zompopo & Defoliador \\
\hline Hymenoptera & Mutillidae & Dasymutilla sp & Hormiga terciopelo & Depredador \\
\hline Hymenoptera & Evaniidae & Prosevaniasp & Avispas & Depredador \\
\hline Hymenoptera & Vespidae & Polistes sp & Catala & Control natural \\
\hline Hymenoptera & Vespidae & Polybiasp & Avispa carnera & Depredador \\
\hline Hymenoptera & Anthophonidae & Xilocopa sp & Abejorro & Hacen orificios \\
\hline Hymenoptera & Apidae & Apis mellifera & Abeja & polinizador \\
\hline Hymenoptera & Apidae & Trigona sp & Congo & Polinizador \\
\hline Hymenoptera & Apidae & Epeolus sp & Abejorro & Polinizador \\
\hline Hymenoptera & Ichneumonidae & Ophion sp & Ichneumonidos & Parasitoides \\
\hline Hymenoptera & Sphecidae & Sceliphron $s p$ & Avispa cintura de hilo & Depredador \\
\hline Hymenoptera & Pompilidae & Pepsis sp & Avispa cazadora de arañas & Depredador \\
\hline Coleoptera & Elateridae & Aeoulus sp & Gusano alambre & Ataca raíces \\
\hline Coleoptera & Elateridae & Conoderus sp & Gusano alambre & Ataca raíces \\
\hline Coleoptera & Scarabaeidae & Phillophaga spp & Escarabajo & Raíces (Las larvas) \\
\hline Coleoptera & Scarabaeidae & Anomala sp & Escarabajo & Raíces (Las larvas) \\
\hline Coleoptera & Scarabaeidae & Cotinis spp & Escarabajo & Raíces (Las larvas) \\
\hline Coleoptera & Scarabaeidae & Euphoria spp & Escarabajo & Raíces (Las larvas) \\
\hline Coleoptera & Scarabaeidae & Gymnetis sp & Escarabajo & Raíces (Las larvas) \\
\hline Coleoptera & Scarabaeidae & Phanaeus sp & Escarabajo & Degradan materia orgánica \\
\hline Coleoptera & Scarabaeidae & Canthon sp & Escarabajo & Degradan materia orgánica \\
\hline Coleoptera & Scarabaeidae & Viridimicus sp & Escarabajo & Plaga del suelo \\
\hline Coleoptera & Cucurlionidae & Cosmopolites sp & Picudo & Se alimenta del cormo y tallos \\
\hline Coleoptera & Cerambycidae & Cyllene sp & Escarabajo & Barrenador de madera \\
\hline Coleoptera & Cerambycidae & Lissonotus sp & Escarabajo & Barrenador de madera \\
\hline Coleoptera & Cerambycidae & Brasilianus sp & Escarabajo & Barrenador de madera \\
\hline Coleoptera & Buprestidae & Chalcophora sp & Escarabajo & Barrenador de madera \\
\hline Coleoptera & Coccinellidae & Cycloneda sanguinea & Mariquita & Depredador \\
\hline Coleoptera & Chrysomelidae & Cerotoma sp & Tortuguillas & Fitófagos \\
\hline Coleoptera & Chrysomelidae & Eumolpus sp & Tortuguillas & Fitófagos \\
\hline Coleoptera & Chrysomelidae & Acalymma trivitattum & Tortuguillas & Fitófagos \\
\hline Coleoptera & Carabidae & Calleida $s p$ & Escarabajo & Depredador \\
\hline Coleoptera & Tenebrionidae & Glyptasida sp & Falso gusano de alambre & Plaga del suelo \\
\hline Coleoptera & Tenebrionidae & Selenophorus sp & Falso gusano de alambre & Plaga del suelo \\
\hline Coleoptera & Tenebrionidae & Blapstinus sp & Falso gusano de alambre & Ataca raíces \\
\hline Coleoptera & Tenebrionidae & Zophobas sp & Falso gusano de alambre & Plaga del suelo \\
\hline Coleoptera & Nitidulidae & Carpophilus spp & Escarabajitos de la savia & Comen frutas descompuestas \\
\hline Coleoptera & Meloidae & Pyrota decorata & Tinajón & Se alimenta de flores \\
\hline Coleoptera & Histeridae & Hololepta sp & Histeridos & $\begin{array}{l}\text { Se alimenta en materia en } \\
\text { descomposición }\end{array}$ \\
\hline Lepidoptera & Noctuidae & Elaphria nucicolora & Gusano soldado & Se alimenta de la cascara de la piña \\
\hline Lepidoptera & Lycaenidae & Thecla basilides & Barrenador del fruto & $\begin{array}{l}\text { Barrena el fruto y se alimenta de la } \\
\text { piña }\end{array}$ \\
\hline Lepidoptera & Nymphalidae & Dynastor sp & Mariposa & Polinizador \\
\hline Lepidoptera & Pyralidae & Diatraea spp & Papalote & Fitófaga, barrenador de tallo \\
\hline Orthoptera & Grillotalpidae & Neocurtilla hexadactyla & Grillo topo & $\begin{array}{l}\text { Cortan los tallos, comen follaje y } \\
\text { raíces de plantas }\end{array}$ \\
\hline
\end{tabular}


Cuadro 1. Contianuación..

\begin{tabular}{|c|c|c|c|c|}
\hline Orthoptera & Grillidae & Gryllus assimilis & Grillo común & Masticador \\
\hline Orthoptera & Acrididae & Orphulella sp & Salta monte & Masticador \\
\hline Orthoptera & Acrididae & Schistocerca $s p$ & Chapulín & Masticador \\
\hline Hemiptera & Reduviidae & Dysdercus obscuratus & Chinche & Depredador \\
\hline Hemiptera & Reduviidae & Reginia cruciata & Chinche & Depredador \\
\hline Hemiptera & Reduviidae & Triatoma dimidiata & Chinche & Se alimenta de sangre \\
\hline Hemiptera & Pentatomidae & Nezara viridula & Chinche & Chupador \\
\hline Hemiptera & Pentatomidae & Euschistus sp & Chinche & Chupador \\
\hline Hemiptera & Pentatomidae & Proxis punctulatus & Chinche & Chupador \\
\hline Hemiptera & Pentatomidae & Loxa viridis & Chinche & Chupador \\
\hline Hemiptera & Pentatomidae & Edessa sp & Chinche & Chupador \\
\hline Hemiptera & Cydnidae & Pangaeus sp & Maya hedionda & Se alimenta de raíces \\
\hline Hemiptera & Coreidae & Leptoglossus zonatus & Chinche pata de hojas & Chupador \\
\hline Hemiptera & Coreidae & Mozena $s p$ & Chinche & Chupador \\
\hline Hemiptera & Lygaecidae & Ligaeus $s p$ & Chinche & Chupador \\
\hline Hemiptera & Largidae & Largus cintus & Chinche & Chupador \\
\hline Hemiptera & Alydidae & Stenocorus $s p$ & Chinche & Chupador \\
\hline Hemiptera & Cercospidae & Aeneolamia $s p$ & Salivita & Chupador \\
\hline Hemiptera & Cicadellidae & Oncometopia $s p$ & Salta hoja & Chupador \\
\hline Hemiptera & Pseudococcidae & Dysmicoccus brevipes & Cochinilla & Chupador \\
\hline Diptera & Calliphoridae & Cochliomya $s p$ & Tórsalo & Se alimenta de sangre \\
\hline Diptera & Muscidae & Musca domestica & Mosca & Se alimenta de materia descompuesta \\
\hline Diptera & Muscidae & Stomoxys calcitrans & Mosca de los establos & Se alimenta de sangre \\
\hline Diptera & Syrphidae & Allograpta $s p$ & Mosca de las flores & Polinizador \\
\hline Diptera & Stratiomyiidae & Hermetia illucens & Mosca soldado & Se alimenta de materia descompuesta \\
\hline Diptera & Tabanidae & Tabanus sp & Mosca & $\begin{array}{l}\text { Se encuentran en materia } \\
\text { descompuesta }\end{array}$ \\
\hline Diptera & Asilidae & Efferia sp & Mosca ladrona & Depredador \\
\hline Diptera & Sarcophagidae & Sarcophaga sp & Mosca & Parasitoide \\
\hline Diptera & Tachinidae & Lespesia $s p$ & Tachinidos & Parasitoides de larvas \\
\hline Diptera & Tephrytidae & Anastrepha obliqua & Mosca de la fruta & Se alimenta de fruta \\
\hline Diptera & Chrysopidae & Chrysoperla sp & León de los afidos & Depredador \\
\hline Isoptera & Termitidae & Heterotermes $s p$ & comején & Se alimenta de madera \\
\hline Odonata & Libellulidae & Libellula $s p$ & Libélula & Depredador \\
\hline Blattodea & Blattellidae & Supella $s p$ & Cucaracha & Plagas doméstica \\
\hline
\end{tabular}

De acuerdo a los insectos identificados por orden, familia y géneros, en lo que corresponde al hábito alimenticio encontramos; once especies de insectos defoliadores, tres especies que se alimentan de frutas, trece especies consideradas plagas del suelo, trece especies succionadores de floemas, seis especies barrenadores de corteza vegetal, cuatro especies polinizadoras, dieciséis especies depredadoras, tres especies de parasitoides, ocho especies degradadores de materia orgánica, una especie que se alimenta de madera, tres especies que se alimentan de sangre, una especie que se alimenta de cormos y tallos, una especie que se alimenta de flores y una considerada plaga doméstica.

Abundancia de insectos. La abundancia de las poblaciones generalmente se expresa en términos de número de individuos por unidad de superficie (larvas por metro, adultos por tallo, etc.). En este estudio la abundancia se midió en número de insectos por finca y números de insectos por familia, el total de insectos encontrados en las seis fincas de piña evaluadas fue de 10.657 especímenes. En cuanto al número de insectos colectados la abundancia fue mayor en la finca San Felipe presentando durante el período de evaluación una cantidad de 2184 insectos (cuadro 2). La abundancia de insectos por familia, Formicidae proporcionó mayor cantidad de insectos presentando con un total de 4321 insectos y la finca que presentó mayor número de insectos de la familia Formicidae fue
Los Ríos con 844 insectos, estos insectos son depredadores por excelencia y viven en simbiosis con insectos de la familia Pseudococcidae. La familia Scarabaeidae es la segunda familia más numerosa con 2617 insectos encontrados, siendo la finca San Felipe la que presentó la mayor cantidad de insectos en el período evaluado con 571 especímenes, durante los muestreos se observaron larvas realizando daños en el sistema radicular de la planta. Entre los principales géneros identificados de esta familia está, Phyllophaga spp, Cotinis spp y Euphoria spp. (cuadro 2) La familia Pseudococcidae, cuya especie más importante en piña es Dysmicoccus brevipes es una de las familias más numerosas encontradas en los muestreos realizados en follaje y frutos con un total de 832 insectos, finca La Perla fue la que mayor cantidad de insectos obtuvo con 174 especímenes. Estos insectos se alimentan succionando la savia de la planta, son vectores del Pineapple Mealybug Wilt-associated virus (PMWaV), conocido como el virus de Wilt, que ocasiona un desecamiento del ápice hacia la base de la hoja y un enrollamiento en el borde de las hojas más afectadas (BANACOL, 2011), es una de las plagas de mayor importancia a nivel comercial en el cultivo, ya que influyen en bajos rendimientos en la producción de piña. La familia Passallidae tuvo frecuente presencia durante el período evaluado encontrándose un total de 573 insectos, en el período evaluado las mayores poblaciones de esta familia de insectos se encontraron en las fincas La Francia con 248 
y La Perla con 174 insectos (cuadro 2), los insectos colectados pertenecen a la especie Passalus sp; sin embargo estos insectos de hábitos nocturnos y polífagos, no son considerados plagas, ya que sus hábitos alimenticios están limitados a alimentarse de materia vegetal en descomposición.

De la familia Elateridae, se colectaron un total de 374 insectos, los que fueron interceptados en trampas de caída libre, en galones con melaza y estados larvales en observaciones realizadas en el suelo, entre las especies encontradas en los muestreos están Aeoulus sp y Conoderus sp., su importancia radica en que es una plaga que en su
Cuadro 3. Riqueza de especies por finca encontrados en el cultivo de la piña entre marzo a septiembre del 2014 en Ticuantepe, utilizando índice de riquezas de especies de Margalef

\begin{tabular}{lcccc}
\hline Fincas & $\begin{array}{c}\text { Especies por fincas } \\
(\mathrm{S})\end{array}$ & $\begin{array}{c}\text { Total de individuos } \\
(\mathrm{N})\end{array}$ & LnN & $\begin{array}{c}\text { Riqueza de especies } \\
\text { S-1/Ln N }\end{array}$ \\
\hline El Edén & 16 & 1499 & 7.31 & 2.05 \\
Los Caleros & 26 & 1728 & 7.45 & 3.36 \\
San Felipe & 29 & 2184 & 7.69 & 3.64 \\
La Francia & 31 & 2085 & 7.64 & 3.93 \\
Las Perlas & 22 & 1461 & 7.29 & 2.88 \\
Los Ríos & 22 & 1700 & 7.44 & 2.82 \\
\hline
\end{tabular}

Cuadro 2. Abundancia total de insectos por familias encontrados en fincas de piñas evaluadas entre marzo a septiembre del 2015

\begin{tabular}{|c|c|c|c|c|c|c|c|}
\hline \multicolumn{8}{|c|}{ Nombre de la Finca evaluadas en el estudio } \\
\hline & El Edén & Los Caleros & San Felipe & La Francia & La Perla & Los Ríos & Total \\
\hline Formicidae & 677 & 668 & 803 & 704 & 625 & 844 & 4321 \\
\hline Scarabaeidae & 333 & 470 & 751 & 418 & 437 & 388 & 2617 \\
\hline Psedococcidae & 133 & 106 & 132 & 148 & 174 & 139 & 832 \\
\hline Passalidae & 82 & 67 & 114 & 248 & 9 & 53 & 573 \\
\hline Elateridae & 3 & 33 & 189 & 121 & 23 & 5 & 374 \\
\hline
\end{tabular}

fase larval su hábito alimenticio es alimentarse de las raíces, debilitando o matando la planta reduciendo la productividad. Las mayores cantidades de insectos de la familia Elateridae se obtuvieron en las fincas San Felipe 189 insectos y La Francia 121 insectos (cuadro 2).

Riqueza de insectos. Riqueza de insectos, es el número de especies por sitio de muestreo, o sea supone una relación entre el número de especies y el número total de individuos, la riqueza es la forma más sencilla de medir la biodiversidad, ya que se basa únicamente en el número de especies presentes, sin tomar en cuenta el valor de importancia de las mismas y la forma ideal de medirla es contar con un inventario completo que nos permita conocer el número total de especies obtenidas por un censo de la comunidad (Moreno, 2001).

En cuanto a la riqueza de insectos encontradas en el cultivo de la piña en el período comprendido de marzo a septiembre del 2014, fue de 51 familias de insectos y la riqueza total de 84 géneros de insectos encontrados, entre las más importantes por el número de géneros están Scarabaeidae (Phillophaga spp, Anomala sp, Cotinis sp, Euphoria spp, Gymnetis sp, Phanaeus sp, Canthon sp y Viridimicus sp), Formicidae (Camponotus sp, Solenopsis sp, Pheidole sp, Ectatomma sp, Atta sp, Atta cephalotes), Pentatomidae (Nezara viridula, Euschistus sp, Proxis punctulatus, Loxa viridis, Edessa sp) Tenebrionidae (Glyptasida sp, Selenophorus sp, Blapstinus sp, Zophobas sp), Cerambycidae (Cyllene sp, Lissonotus sp, Brasilianus sp), Chrysomelidae (Cerotoma sp, Eumolpus sp, Acalymma trivitattum) y Reduvidae (Dysdercus obscuratus, Reginia cruciata, Triatoma dimidiata) (Borror y De Long, 1979, Hernández Ortiz, 1992, King y Sanders, 1984, Maes, 2004, Jiménez- Martínez y RodriguezFlores, 2014, Nunes y Dávila, 2004, Sáenz y De La Llana, 1990, Nájera y Souza, 2010).
Margalef establece que valores inferiores a 2,0 están relacionados con zonas de baja biodiversidad o riqueza y valores superiores a 5,0 son considerados como indicativos de alta biodiversidad o riqueza (Moreno, 2001). De acuerdo a lo presentado en el (Cuadro 5), los resultados demuestran índices de riquezas normales para todas las fincas, La Francia obtuvo el mayor índice con 3.93, y la finca con el menor índice resulto ser El Edén con 2.05.

Índice de diversidad de las principales familias de insectos asociados al cultivo de la piña. Con los datos obtenidos en las diferentes fechas de muestreos durante los meses de marzo a septiembre del 2014 en las fincas: El Edén, Los Caleros, San Felipe, La Francia, La Perla y Los Ríos, se calculó la diversidad, obtenida por medio del índice de diversidad Shannon-Weaver (cuadro 4).

Los valores de diversidad media (según Shannon Weaver) para las fincas evaluadas son bajos ya que oscilan generalmente entre 1.01 y 1.44 , la familia con mayor índice fue Formicidae con 1.44 obtenido en las fincas Los Caleros, Las Perlas, San Felipe y La Francia, esta misma familia obtuvo un índice de 1.43 en la finca El Edén. Otra familia con alto índice de biodiversidad fue Scarabaeidae con 1.43 en la finca Las Perlas. Los índices de diversidad más bajos fueron de 1.01 en la finca El Edén y 1.02 en la finca Los Ríos para las Familias Elateridae y Passalidae con 1.03 en la finca Las Perlas. Al realizar una comparación de estos datos obtenidos en los muestreos, se puede decir que la diversidad se manifestó bastante homogénea en todas las fincas. 
Cuadro 4. Índice de diversidad Shannon-Weaver de las principales familias de insectos asociados al cultivo del Piña en Ticuantepe, entre marzo y septiembre del 2014

Familia de insectos Índice de diversidad de Shannon-Weaver

\begin{tabular}{lcccccc}
\cline { 2 - 6 } & Los Caleros & Las Perlas & San Felipe & La Francia & Los Ríos & El Edén \\
\hline Formicidae & 1.44 & 1.44 & 1.44 & 1.44 & 1.42 & 1.43 \\
Scarabaeidae & 1.42 & 1.43 & 1.42 & 1.38 & 1.40 & 1.40 \\
Pseudococcidae & 1.19 & 1.29 & 1.19 & 1.21 & 1.23 & 1.24 \\
Passalidae & 1.13 & 1.03 & 1.17 & 1.29 & 1.11 & 1.18 \\
Elateridae & 1.08 & 1.07 & 1.24 & 1.18 & 1.02 & 1.01 \\
\hline
\end{tabular}

Fluctuación poblacional de insectos de la familia Formicidae, Scarabaeidae, Pseudococcidae, Passallidae y Elateridae. En la evaluación realizada a las fincas Los Caleros, Las Perlas, San Felipe, La Francia, Los Ríos y El Edén, se comparó la fluctuación poblacional de insectos de la familia Formicidae, Scarabaeidae, Pseudococcidae, Passallidae y Elateridae por fecha de muestreo. Al realizar el análisis de varianza del número de insectos de la familia Formicidae encontrado en 21 muestreos en las fincas evaluadas, se encontró diferencia significativa en la cantidad de insectos encontrados con probabilidad de $(P=0.0001)$ (Cuadro 5), el mayor número de estos insectos lo presentaron las fincas San Felipe y Los Ríos con un promedio de 4.39 y 4.28 insectos respectivamente. Algunas especies de la familia Formicidae que comúnmente se encontraron en el cultivo de la piña están: Solenopsis sp, Pheidole sp y Camponotus sp. Esta familia de insectos tienen un tamaño de pequeño a grande (1-30mm), cuerpo delgado, antenas con 6-13 segmentos (Sáenz y De la Llana, 1990, Nájera y Souza, 2010, Urías., et al, 2007), el color de estos insectos generalmente es bronceado, café o negro, estos insectos se desarrollan por metamorfosis completa, las hormigas son insectos sociales que viven en colonias que tienen una o más reinas y muchas obreras (Jiménez- Martínez, 2009). En cuanto al hábito alimenticio, la familia Formicidae son defoliadores y depredadores por excelencia; sin embargo en el cultivo de la piña, el hábito de estos insectos, no tiene mucha importancia para considerarla como plagas; más bien estos insectos en el cultivo de la piña desarrollan una actividad depredadora para unas especies de insectos y de simbiosis con insectos de la familia Pseudococcidae.

Al realizar el análisis de varianza del número de insectos de la familia Scarabaeidae encontrado en 21 muestreos realizados en las fincas evaluadas, se encontró diferencia significativa en la cantidad de insectos encontrados con probabilidad de $(p=0.0009)$ (Cuadro 5), el mayor número de estos insectos lo presentaron las fincas San Felipe y Las Perlas con un promedio de 4.39 y 3.67 insectos respectivamente. Los adultos de esta familia de insectos son considerados inofensivos, vuelan de noche y son atraídos por la luz, su hábito alimenticio es de alimentarse de azucares y son atraídos por las flores ricas en néctar, siendo el estado larval el de más importancia debido a que son fitófagos, masticadores, barrenadores, cortadores, minadores, tejedores y algunos formadores de agallas (Sáenz y de la Llana, 1990. Jiménez-Martínez, 2009). Estos insectos son plagas de cultivos. Su hábito alimenticio, las larvas se alimentan de raíces y algunos adultos del follaje y flores de sus hospederos y un número considerable de especies son benéficas degradando materia orgánica (Sáenz y De la Llana, 1990). Al realizar el análisis varianza del número de insectos de la familia Pseudococcidae encontrado en 21 muestreos realizados en las fincas evaluadas, no se encontró diferencia significativa en la cantidad de insectos encontrados con probabilidad $(p=0.0631)$ (cuadro 5), el mayor número de estos insectos lo presentaron las fincas Las Perlas y Los Ríos con un promedio de 3.78 y 3.31 insectos respectivamente. La especie más representativa de esta familia y de mucha importancia en el cultivo de la piña es Dysmicoccus brevipes, reconocida por transmitir la enfermedad de la marchitez de la piña, ataca durante todo el ciclo del cultivo, su hábito alimenticio es succionar la savia de raíces, hojas y cavidades florales del fruto, presenta una amplia diversidad de hospederos alternos en la zona entre ellos: banano, plátano, plantas ornamentales, cyperáceas y algunos pastos.

Los Pseudococcidae tienen una longitud entre 1 a 8 $\mathrm{mm}$, cuerpo aplanado, oval y elongado o hemisférico tienen el cuerpo cubierto de secreciones, presentan dimorfismo sexual, las hembras son ápteras los machos son alados (Nunes y Dávila, 2004, Jimenez-Martínez, 2009). El período más crítico de incidencia de Dysmicoccus brevipes, se concentra entre la sexta y doceava semana de floración, donde los “ojos de la piña" se mantienen abiertos a la entrada de la plaga. El control de esta plaga resulta muy difícil, debido a que puede afectar en cualquier etapa de desarrollo del cultivo. Al realizar el análisis de varianza del número de insectos de la familia Passalidae encontrado en 21 muestreos realizados en las fincas evaluadas, da como resultado diferencia significativa en la cantidad de insectos encontrados con probabilidad de ( $p$ $=0.0001$ ) (cuadro 5), el mayor número de estos insectos lo presentaron las fincas La Francia y El Edén con un promedio de 4.96 y 3.42 insectos respectivamente. La familia Passallidae es reconocida por su hábito alimenticio saproxilófago; es decir, se alimenta de los troncos podridos, muertos y en descomposición, mismos que se localizan sobre el suelo, por lo que reciclan el carbono atrapado en la madera y lo depositan en el suelo por medio de sus excretas, abonando, engrosando y enriqueciendo los suelos donde estos escarabajos habitan (Nunes y Dávila, 2004). Al realizar el análisis de varianza del número de insectos de la familia Elateridae encontrado en 21 muestreos realizados en las fincas evaluadas, se encontró diferencia significativa en la cantidad de insectos encontrados con probabilidad de $(p=0.0206)$ (cuadro 5), el mayor número de estos insectos lo presentaron las fincas San Felipe y La Francia con un promedio de 9.45 y 4.03 insectos respectivamente. Los Elateridae son escarabajos de tamaño pequeño a grande $(2-100 \mathrm{~mm})$, con cuerpo alargado, más o menos aplanados; abdomen puntiagudo, antenas con $11 \mathrm{seg}$ 
Cuadro 5. Número de insectos de la familia Formicidae, Scarabaeidae, Pseudococcidae, Passallidae y Elateridae encontrado en 21 muestreos en el cultivo de la piña en seis fincas evaluadas, entre marzo y septiembre del 2014 en Ticuantepe

\begin{tabular}{lccccc}
\hline Familia & Formicidae & Scarabaeidae & Pseudococcidae & Passallidae & Elateridae \\
\hline Finca & Media $\pm \mathrm{ES}$ & Media \pm ES & Media $\pm \mathrm{ES}$ & Media $\pm \mathrm{ES}$ & Media \pm ES \\
\hline San Felipe & $4.39 \pm 0.19 \mathrm{a}$ & $4.39 \pm 0.28 \mathrm{a}$ & $3.78 \pm 0.54$ & $4.96 \pm 0.36 \mathrm{a}$ & $9.45 \pm 3.12 \mathrm{a}$ \\
Los Ríos & $4.28 \pm 0.29 \mathrm{a}$ & $3.67 \pm 0.27 \mathrm{~b}$ & $3.31 \pm 0.32$ & $3.42 \pm 0.48 \mathrm{a}$ & $4.03 \pm 0.56 \mathrm{~b}$ \\
La Francia & $3.71 \pm 0.17 \mathrm{~b}$ & $3.62 \pm 0.436 \mathrm{~b}$ & $3.29 \pm 0.31$ & $3.26 \pm 0.31 \mathrm{~b}$ & $3.30 \pm 0.76 \mathrm{~b}$ \\
Los Caleros & $3.41 \pm 0.17 \mathrm{~b}$ & $3.40 \pm 0.34 \mathrm{~b}$ & $2.77 \pm 0.31$ & $2.94 \pm 0.51 \mathrm{~b}$ & $1.67 \pm 0.33 \mathrm{c}$ \\
Las Perlas & $3.36 \pm 0.18 \mathrm{~b}$ & $3.12 \pm 0.201 \mathrm{c}$ & $2.59 \pm 0.21$ & $2.25 \pm 0.75 \mathrm{~b}$ & $1.50 \pm 0.50 \mathrm{c}$ \\
El Edén & $3.35 \pm 0.15 \mathrm{~b}$ & $2.62 \pm 0.16 \mathrm{c}$ & $2.52 \pm 0.27$ & $2.09 \pm 0.17 \mathrm{~b}$ & $1.28 \pm 0.14 \mathrm{c}$ \\
SD; CV & $2.73 ; 72.80$ & $3.29 ; 94.75$ & $2.30 ; 75.84$ & $2.07 ; 58.98$ & $7.21 ; 159.97$ \\
$p$ & $0.0001 \mathrm{DS}$ & $0.0009 \mathrm{DS}$ & $0.0631 \mathrm{NS}$ & $0.0001 \mathrm{DS}$ & $0.0206 \mathrm{DS}$ \\
df; ; F & $1148 ; 1153 ; 5.77$ & $748 ; 753 ; 4.19$ & $268 ; 273 ; 2.12$ & $157 ; 162 ; 8.55$ & $77 ; 82 ; 2.85$ \\
\hline
\end{tabular}

ES: Error estándar de la media, CV: Coeficiente de variación, SD: Desviación Estándar, p: Probabilidad según Duncan, F: Fisher calculado, df: grados de libertad del error, n: Número de datos utilizados, DS: Diferencia Significativa, *: Medias con letras distintas existe diferencia significativa.

mentos, filiformes, aserradas, o pectinadas (Sáenz y De la Llana, 1990). Las larvas son alargadas y cilíndricas del tipo elateriforme, conocidas como gusanos de alambre, estas son rígidas y de color amarillo a café claro, viven en el suelo y en madera descompuesta (Nunes y Dávila, 2004).

El hábito alimenticio observado de esta familia, es que en estado larvario se alimenta de las raíces y cuello de las plantas, pudiendo producirse la muerte en plantas jóvenes. En plantas adultas provocan debilitamiento generalizado de la planta al cortar la circulación de la savia, marchitez y retraso en el crecimiento y de manera indirecta las heridas provocadas en el tallo son vías de entrada de agentes patógenos (Nunes y Dávila, 2004).

\section{CONCLUSIONES}

Las principales familias de insectos asociados al cultivo de la piña fueron Formicidae, Scarabaeidae, Pseudococcidae, Passallidae y Elateridae.

La familia de insecto que obtuvo el mayor índice de diversidad en las fincas de piña fue Formicidae y la más baja fue Elateridae.
Las familias de insectos más abundantes en piña y encontradas en el estudio fueron Formícidae y Scarabaeidae, y las fincas piñeras donde se encontró mayor abundancia de insectos fueron Los Ríos y San Felipe.

De acuerdo la fluctuación poblacional, las mayores poblacionales de insectos se alcanzaron en los meses de abril, mayo y julio y las familias de insectos que presentaron mayor incidencia fueron; Formicidae, Scarabaeidae y Pseudococcidae.

Los principales hábitos alimenticios de las especies insectiles asociados al cultivo de la piña fueron defoliadores, plagas del suelo, succionadores de floemas, barrenadores de corteza vegetal, polinizadoras, depredadores, parasitoides y degradadores de materia orgánica.

\section{AGRADECIMIENTO}

Los autores de esta investigación agradecen a la Universidad Nacional Agraria (UNA), al Instituto de Protección y Sanidad Agropecuaria (IPSA) y al Departamento de Agricultura de los Estados Unidos (USDA) por la financiación económica de este estudio.

\section{REFERENCIAS BIBLIOGRÁFICAS}

ATC (Asociación de Trabajadores del Campo, NI), Escuela Obrera Campesina Internacional Francisco Morazán.2011. Manejo y Producción de Piña con Enfoque agro ecológico. En línea. s.e. Managua. NI. Consultado 19 oct. 2013. Disponible en: http://www. escampi.org/PDF/Manejo_Agronomico_Pina.pdf

Barrios Diaz, B; Alatorre Rosas, R; Calyecac Cortero, HG; Bautista Martinez, N. 2004. Identificación y fluctuación poblacional de la plagas de la col (Brassica oleracea var. Capitata) y sus enemigos naturales en Acatzingo, Puebla, MX. Agrociencia 38: 339 - 248.

Bonilla, L. 1992. Cultivo de piña, boletín técnico No. 11. Serie cultivos, Fundación de desarrollo agropecuario, INC. Santo Domingo, República Dominicana. 24 pp.

Borror, D; De Long, D; Triplehorn, C. 1979. An introduction to the study of insects. 5 ed. Philadelhia, US. 928 p.

FDA (Fundación de Desarrollo Agropecuario, INC. RD). 1992. El Cultivo de la piña. En línea. Ed. PP, Peña. Santo Domingo, RD. Consultado 17 sep. 2013. Boletín técnico No 11. Disponible en http://www.rediaf.net.do/publicaciones/guias/download/pina.pdf

Hernández Ortiz, V. 1992. El Género Anastrepha spp (Schiner 1868), en México (Diptera: Tephritidae). Taxonomía, distribución y sus plantas huéspedes. Instituto de Ecología, Sociedad Mexicana de Entomologia. MX. 161 p. 
INIFOM (Instituto Nicaragüense de Fomento Municipal). 2009. Ficha técnica del Municipio de Ticuantepe. En línea. s.e. Consultado 19 sep. 2013 Disponible en: http://www.inifom.gob.ni/municipios/documentos/MANAGUA/ticuantepe.pdf

INTA (Instituto Nicaragüense de Tecnología Agrícola), 2001.Cultivo de la piña. Ed. H. Obregón. Managua NI. 24 p. (Guía Tecnológica no.7).

Jiménez Martínez, E; Rodriguez Flores, O. 2014. Universidad Nacional Agraria. Insectos: Plagas de cultivos en Nicaragua. Managua, NI. 218 p.

Jiménez Martínez, E; 2009. Entomología. Universidad Nacional Agraria (UNA). Dirección de Investigación Extensión y Posgrado (DIEP). Editronic, se. Managua, NI. 112 p.

King, A; Sanders, J. 1984. Las plagas invertebradas de cultivos anuales alimenticios en America Central. CATIE. Turrialba, Costa Rica. CR. 182 p.

Lacayo, R; Mayorga, J. 2014. Abundancia, riqueza y diversidad insectil asociada al cultivo de Marango (Moringa oleifera L.). Ingeniero agrónomo. Managua, NI. Universidad Nacional Agraria. 56 p.

Maes, JM. 2004. Insectos asociados a algunos cultivos tropicales en el atlántico de Nicaragua. Parte XIII. Piña (Ananas comosus, Bromeliaceae). En línea. León, NI. s.e. Consultado 20 sep. 2013. Disponible en: http://www.bio-nica.info/RevNicaEntomo/ 64-2004-S1-24.pdf

Margalef, DR. 1958. Information theory in ecology. General systematics, 3: 36-71.

Moreno, C. 2001. Métodos para medir la biodiversidad. CYTED, ORCIT/UNESCO \& SEA. Zaragoza, España. ES. M\&T - Manuales y Tesis SEA. vol 1.84 p.

Nájera, M; Souza, B. 2010. Insectos benéficos. Guía para su identificación. INIFAP. 1 ed. Michoacán, MX. 73 p.

Nunes, C; Dávila, ML. 2004. Taxonomía de las principales familias y subfamilias de insectos de interés agrícolas en Nicaragua. UCAPSE (Universidad Católica Agropecuaria del Trópico Seco Estelí) Nicaragua. p 164.

OIRSA (Organismo Internancional Regional de Sanidad Agropecuaria). 1999. Manual técnico buenas prácticas de cultivo en piña. $44 \mathrm{p}$.

Rugama Lovo, IM, López- Vílchez, ME. 2011. Identificación y descripción de los principales insectos rastreros asociados al cultivo del maranón (Anacardium occidentalis L.) orgánico y convencional, en león, Nicaragua. Ingeniero agrónomo. Managua, NI. Universidad Nacional Agraria. 94 p.

Sáenz, M; De La Llana, A. 1990. Entomología sistemática. UNA (Universidad Nacional Agraria). Managua, NI. 225 p.

SAS Institute. 2003. SAS Languaje guide for personal computer. University of Nebraska. Cary, NC, USA.V.91

Shannon, CE; Weaver, W. 1949. The mathematical theory of communication, Urbana, Illinois. University of Illinois Press, 117 p.

Somarriba, E. 1999. Diversidad Shannon. En línea. Agroforestería en las Américas. 6 (23): 72-74. Consultado 26 nov. 2013 . Disponible en http://orton.catie.ac.cr/repdoc/A3377E/A3377E.PDF

Téllez, M; Jirón, V. 2014. Identificación y variación poblacional de insectos asociados al cultivo de marango (Moringa oleifera L.) en Managua, Nicaragua durante los meses de noviembre 2012 a abril 2013. Tesis Ingeniero Agrónomo. UNA. 124 p.

Urías López, MA; Salazar Garcia, S; Johandsen Naime, R. 2007. Identificacion y fluctuación poblacional de especies de trips (Thysanoptera) en aguacate Hass en Nayarit, México. MX. Revista Chapingo Serie Horticultura 13(1): 49-54.

Vásquez, LL; Matienzo, Y; Simonetti, A; Moreno, D; Álvarez, A. 2009. Diversidad de especies de hormigas (Hymenoptera: Formicidae) en cafetales afectados por Hypothenemus hampei Ferrari (Coleoptera: Cucurlionidae: Escolytinae). Ciudad de La Habana, Cuba. CU. Fitosanidad. 13(3):163-168. 